\title{
A Complex-Baseband Active-Set Approach for Tone Reservation PAR Reduction in OFDM Systems
}

\author{
Meng Wang, Daniel E. Quevedo, Graham C. Goodwin \\ School of Electrical Engineering \& Computer Science \\ The University of Newcastle \\ Callaghan, NSW 2308, Australia \\ MWang@studentmail.newcastle.edu.au,dquevedo@ieee.org, \\ Graham.Goodwin@newcastle.edu.au
}

\author{
Brian S. Krongold \\ ARC Special Research Centre for \\ Ultra-Broadband Communications \\ The University of Melbourne \\ Parkville, VIC 3010, Australia \\ bsk@ee.unimelb.edu.au
}

\begin{abstract}
In this paper, we present an active-set approach for peak-to-average power ratio (PAR) reduction via tone reservation in complex-baseband orthogonal frequency division multiplexing (OFDM) systems. In the complex-baseband model, the optimization problem of tone reservation turns out to be a quadratically constrained quadratic program (QCQP), which is computationally prohibitive for a practical implementation. To address this problem, we consider the complex-valued samples of an OFDM symbol as vectors in the complex plane and develop an iterative clipping algorithm. In our approach, PAR reduction is achieved by minimizing the radius of the circle that encloses all samples, thus minimizing the maximum power peak. Simulation results show that the proposed algorithm can achieve near-optimal performance with fast convergence and limited complexity.
\end{abstract}

\section{INTRODUCTION}

Orthogonal frequency division multiplexing (OFDM) techniques [1] have been widely recognized as a promising solution for broadband wireless communication. Due to its high spectral efficiency and robustness to multipath fading environments, OFDM has been adopted in a number of applications such as wireless local area network (WLAN) standards including IEEE 802.11a [2] and European HIPERLAN/2 [3] and, more recently, the IEEE 802.16e mobile worldwide interoperability for microwave access (WiMAX) standard [4].

One major drawback of OFDM is the high peak-to-average power ratio (PAR), which leads to low power efficiency and other design challenges for its implementation. In the literature, various PAR reduction techniques have been proposed to deal with the high PAR nature of OFDM signals (see the overview paper [5] and the references therein). One such technique is the tone reservation method [6]. The method constructs an additive PAR reduction signal that uses a small subset of subcarriers (reserved tones). These reserved tones are orthogonal with the data tones and, hence, cause no distortion. The PAR reduction signal is optimized over the space of reserved tones for minimizing the PAR level.

Tellado's work [6] introduced the principle of tone reservation and cast the optimization problem of PAR reduction as a linear program (LP) and a quadratically constrained quadratic program (QCQP) for the real-baseband case and complexbaseband case, respectively. Finding the exact solution to LP or QCQP is computationally challenging for implementation. Thus, [6] also proposed two suboptimal algorithms with less complexity. However, the suboptimal algorithms suffer from slow convergence and the fact that they require choosing the predetermined clipping level. A fast-converging algorithm for tone reservation was proposed in [7] based on the activeset method. This algorithm was developed for real-baseband OFDM systems, but also can be extended to the complexbaseband case by using a LP-based approximation, albeit leading to extra computational burden and degradation of performance. To handle the complex-baseband case, a subgradientbased algorithm was proposed in [8], which focused on solving a general min-max optimization problem. The drawbacks of this algorithm are slow convergence and that the parameters used in the subgradient algorithm are difficult to determine such that the optimal performance can be achieved.

In the present work, we extend the active-set strategy proposed in [7] to complex-baseband OFDM. In complexbaseband systems, the task of PAR reduction is equivalent to minimizing the radius of the circle that encloses all samples in the complex plane. We propose an iterative algorithm in which the power peaks are clipped iteratively by a set of complex impulse-like signals generated by the reserved tones. In each iteration, our algorithm finds an optimal combination of these signals such that all balanced peaks can be reduced by identical magnitude, thereby avoiding peak regrowth. The proposed algorithm converges quickly and gives a PAR level that is close to the optimal (though difficult to compute) solution. The approach incurs only limited computational cost and can therefore be regarded as a PAR reduction solution in practical OFDM and uplink OFDMA systems [9].

\section{SySTEM MODEL AND THE TONE RESERVATION METHOD}

Consider a complex-baseband OFDM system with $N$ subcarriers indexed by $k=0,1, \ldots, N-1$, see, e.g., [6,7]. Data bits are mapped into a complex-valued quadrature amplitude modulation (QAM) symbol modulated on the $k$ th subcarrier, denoted by $X_{k}$. The time-domain samples of the complexbaseband signal can be obtained by the inverse fast Fourier 
transform (IFFT), i.e.,

$$
x_{n}=\frac{1}{\sqrt{N}} \sum_{k=0}^{N-1} X_{k} e^{\frac{j 2 \pi n k}{N L}}, \quad 0 \leq n \leq N L-1
$$

where $L$ is the oversampling factor. Note that $x_{n}$ is the sum of many symbols modulated on different subcarriers. Thus, if the number of subcarriers $N$ is large enough, time-domain samples $\mathbf{x}=\left\{x_{n}\right\}$ are approximately complex Gaussian random variables according to the central limit theorem. The PAR of the corresponding OFDM signal is given by

$$
\operatorname{PAR}\{\mathbf{x}\}=\frac{\|\mathbf{x}\|_{\infty}^{2}}{E\left\{\left|x_{n}\right|^{2}\right\}}
$$

where $E\left\{\left|x_{n}\right|^{2}\right\}$ denotes the average power of the signal.

In the tone reservation method, $N_{r}$ reserved tones are used to construct a time-domain signal $\mathbf{c}=\left\{c_{n}\right\}$ that is added to the original signal to compensate the power peaks via

$$
\begin{aligned}
\bar{x}_{n} & =x_{n}+c_{n} \\
& =\frac{1}{\sqrt{N}} \sum_{k=0}^{N-1}\left(X_{k}+C_{k}\right) e^{\frac{j 2 \pi n k}{N L}}
\end{aligned}
$$

where $\mathbf{X}=\left\{X_{k}\right\}$ and $\mathbf{C}=\left\{C_{k}\right\}$ denote the signals transmitted on the data tones and reserved tones, respectively. To avoid signal distortion, $\mathbf{X}$ and $\mathbf{C}$ are orthogonal with each other, i.e.,

$$
X_{k}+C_{k}= \begin{cases}X_{k}, & \text { if } k \notin R \\ C_{k}, & \text { if } k \in R\end{cases}
$$

where $R$ denotes the index set of reserved tones. After the PAR reduction is applied, the resultant PAR is given by

$$
\operatorname{PAR}\{\overline{\mathbf{x}}\}=\frac{\left\|\mathbf{x}+Q_{c} \mathbf{C}\right\|_{\infty}^{2}}{E\left\{\left|x_{n}\right|^{2}\right\}}
$$

where $Q_{c}$ is the submatrix of the IFFT matrix

$$
Q \triangleq \frac{1}{\sqrt{N}}\left(\begin{array}{cccc}
1 & 1 & \cdots & 1 \\
1 & e^{j 2 \pi \frac{1 \times 1}{N L}} & \cdots & e^{j 2 \pi \frac{1 \times(N L-1)}{N L}} \\
\vdots & \vdots & \ddots & \vdots \\
1 & e^{j 2 \pi \frac{(N L-1) \times 1}{N L}} & \cdots & e^{j 2 \pi \frac{(N L-1) \times(N L-1)}{N L}}
\end{array}\right)
$$

obtained by selecting the columns according to the set of reserved tones. The problem of minimizing PAR is then equivalent to computing a complex vector $\mathbf{C} \in \mathbb{C}^{N_{r} \times 1}$, which minimizes the maximum peak of the time-domain samples. This amounts to finding:

$$
\hat{\mathbf{C}}=\arg \min _{\mathbf{C}}\left\|\mathbf{x}+Q_{c} \mathbf{C}\right\|_{\infty}
$$

Note that the optimization problem in (7) is essentially a special case of a QCQP [6]

$$
\begin{aligned}
& \min _{\mathbf{C}} t \\
& \text { subject to: }\left|x_{n}+q_{n} \mathbf{C}\right|^{2} \leq t
\end{aligned}
$$

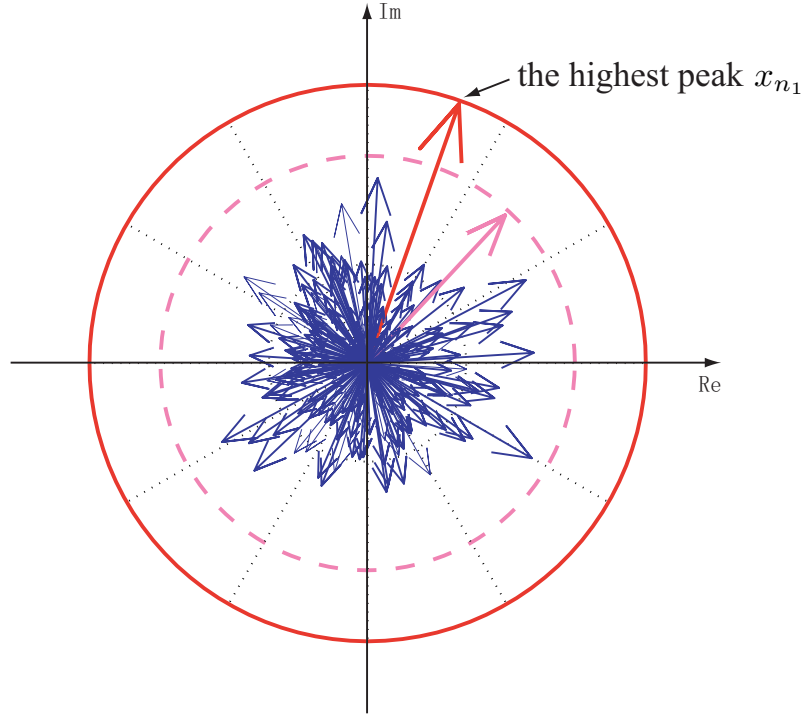

Fig. 1. The time-domain samples of an OFDM symbol in the complex plane.

for $n=0, \ldots, N-1$, where $q_{n}$ is the $n$th row of $Q_{c}$. Since it is computationally expensive to solve the QCQP, the need to develop low-complexity fast-converging algorithms for complex-baseband tone reservation arises. In the next section, we will present such a scheme.

\section{A SIMPLE ALGORITHM FOR TONE RESERVATION}

For a complex-baseband OFDM system, as described in the previous section, we regard the complex-valued time-domain samples of an OFDM symbol as $N L$ vectors in the complex plane, as illustrated in Fig.1. Both real part and imaginary part of each sample need to be considered for calculating its power or equivalently, its distance to the origin. PAR reduction amounts to finding an optimal $\mathbf{C}$ such that the radius of the circle that encloses all the compensated samples $\mathbf{x}+Q_{c} \mathbf{C}$ is minimized in the complex plane.

\section{A. Peak Clipping in the Complex Plane}

To obtain a small covering circle in the complex plane, the simplest method is clipping the power peaks with the PAR reduction signal generated by reserved tones. Ideally, one would like to clip a particular peak by constructing a compensation vector, say $\mathbf{c}^{\text {ideal, }}$ that points to the opposite direction of that peak. Then the time-domain signal would be written as

$$
\overline{\mathbf{x}}=\mathbf{x}+\mathbf{c}^{\text {ideal }}=\mathbf{x}+\sum_{n \in A} \alpha_{n} \mathbf{p}_{n}^{\text {ideal }}
$$

where $A$ is the index set of clipped samples and $\alpha_{n}$ is the real-valued clipping amplitude. In (9), $\mathbf{p}_{n}^{\text {ideal }}$ denotes the ideal clipping impulse corresponding to peak at location $n$, as shown in Fig.2(a). It can be defined as

$$
\mathbf{p}_{n}^{\text {ideal }}=-\frac{x_{n}}{\left|x_{n}\right|} \mathbf{e}_{n}
$$




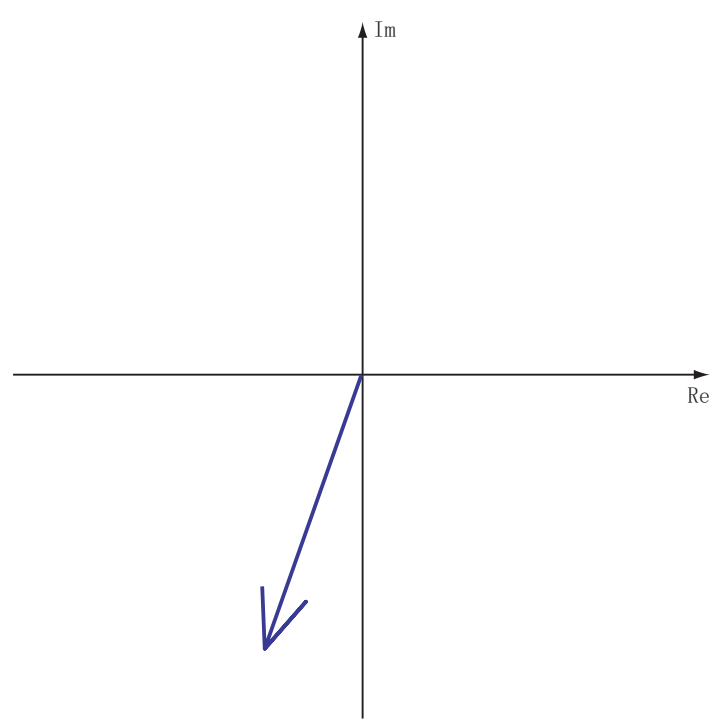

(a) The ideal clipping impulse $\mathbf{p}_{n}^{\text {ideal }}$ at location $n$.

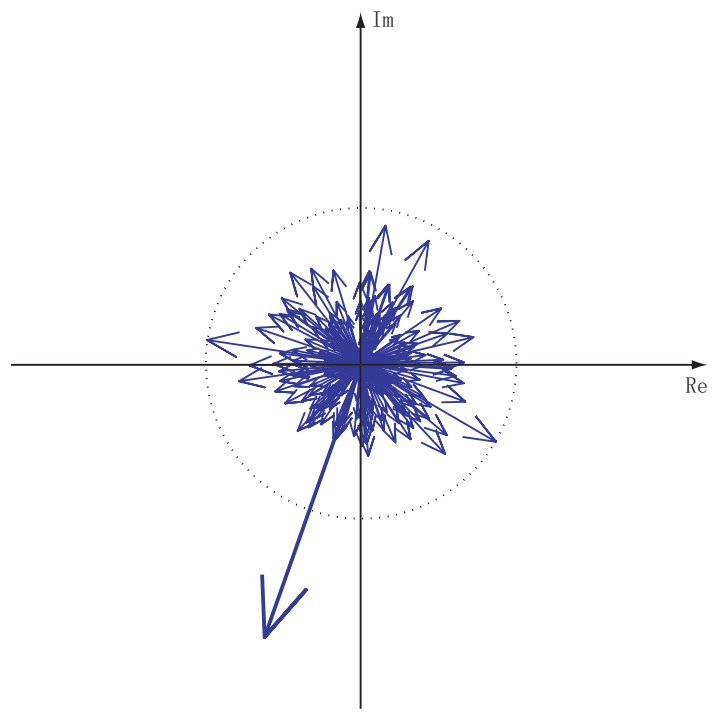

(b) The approximate impulse $\mathbf{p}_{n}$ at location $n$

Fig. 2. The ideal impulse $\mathbf{p}_{n}^{\text {ideal }}$ and its approximate impulse $\mathbf{p}_{n}$ corresponding to the $n$th sample.

where $\mathbf{e}_{n}$ refers to a discrete-time impulse, i.e.,

$$
\mathbf{e}_{n}=(\underbrace{0, \cdots, 0}_{n-1}, 1,0, \cdots, 0)^{T}
$$

where $(\cdot)^{T}$ denotes transpose.

In general, however, the ideal clipping impulse $\mathbf{p}_{n}^{\text {ideal }}$ cannot be generated by the reserved tones since the FFT of a discretetime impulse spreads over all subcarriers in the frequencydomain. To achieve PAR reduction, we will focus on generating a complex-valued approximate impulse, say $\mathbf{p}_{n}$, by minimizing the mean square error between $\mathbf{p}_{n}$ and $\mathbf{p}_{n}^{\text {ideal }}$, i.e.,

$$
\mathbf{p}_{n}=\arg \min \left\|\mathbf{p}_{n}-\mathbf{p}_{n}^{\text {ideal }}\right\|_{2}=\arg \min \left\|Q_{c} \mathbf{P}_{n}-\mathbf{p}_{n}^{\text {ideal }}\right\|_{2}
$$

where $\mathbf{P}_{n}$ is the FFT of $\mathbf{p}_{n}$, that is, the corresponding signal transmitted on the reserved tones. Essentially, $\mathbf{p}_{n}$ is the projection of the ideal impulse $\mathbf{p}_{n}^{\text {ideal }}$ onto the space generated by the reserved tones. The solution of (12) is given by

$$
\mathbf{P}_{n}=\left(Q_{c}^{H} Q_{c}\right)^{-1} Q_{c}^{H} \mathbf{p}_{n}^{\text {ideal }}
$$

where $(\cdot)^{H}$ denotes conjugate transpose. Note that

$$
Q_{c}^{H} Q_{c}=L \cdot \mathbf{I}_{N_{r}}
$$

where $\mathbf{I}_{N_{r}}$ is an $I_{r} \times I_{r}$ identity matrix. Thus, $\mathbf{P}_{n}$ can be written as

$$
\mathbf{P}_{n}=-\frac{x_{n}}{L\left|x_{n}\right|} q_{n}^{H}
$$

where $q_{n}$ is the $n$th row of $Q_{c}$. The corresponding time-domain approximate impulse is given by

$$
\mathbf{p}_{n}=-\frac{x_{n}}{L\left|x_{n}\right|} Q_{c} q_{n}^{H}
$$

For a given set of peaks $A$, with the linearity of FFT and (9), the PAR reduction signal to be transmitted on the reserved tones can be written as

$$
\mathbf{C}=\sum_{n \in A} \alpha_{n} \mathbf{P}_{n}
$$

In this approximate case, however, it is difficult to determine $\alpha_{n}$ directly. As shown in Fig.2(b), the approximate impulse $\mathbf{p}_{n}$ has a cloud of non-zero vectors over many time-domain samples. This fact means that clipping one peak might increase the peaks of other samples. To handle this problem, we propose an iterative clipping algorithm in the complex plane, to be described in the sequel.

\section{B. An Iterative Clipping Algorithm in the Complex Plane}

As illustrated in Fig.1, all samples should be bounded in the circle in the process of clipping. To guarantee this, the proposed algorithm can be described as follows ${ }^{1}$ :

1) Begin with the highest peak $x_{n_{1}}$ whose magnitude is equal to the radius of the circle.

2) Clip $x_{n_{1}}$ with the corresponding approximate impulse $\mathbf{p}_{n_{1}}$ until it is balanced with another peak. These two peaks have the same magnitude corresponding to the current radius. See Fig.3.

3) Clip the two balanced peaks obtained in step 2) in a direction, say the clipping direction. Along this direction, the magnitude of both peaks can be reduced identically until both of them are balanced with a new peak. Now these three peaks have the same magnitude, corresponding to the new radius.

4) At the $i$ th iteration, $i$ balanced peaks are clipped equally until a new peak reaches the circle. Repeat this process until the radius of the circle $r^{(i)}$ cannot be reduced further significantly. As will become apparent, only few iterations are needed, see Section IV.

${ }^{1}$ Note that the proposed algorithm extends the active-set strategy adopted in [7] to the complex-baseband model. 


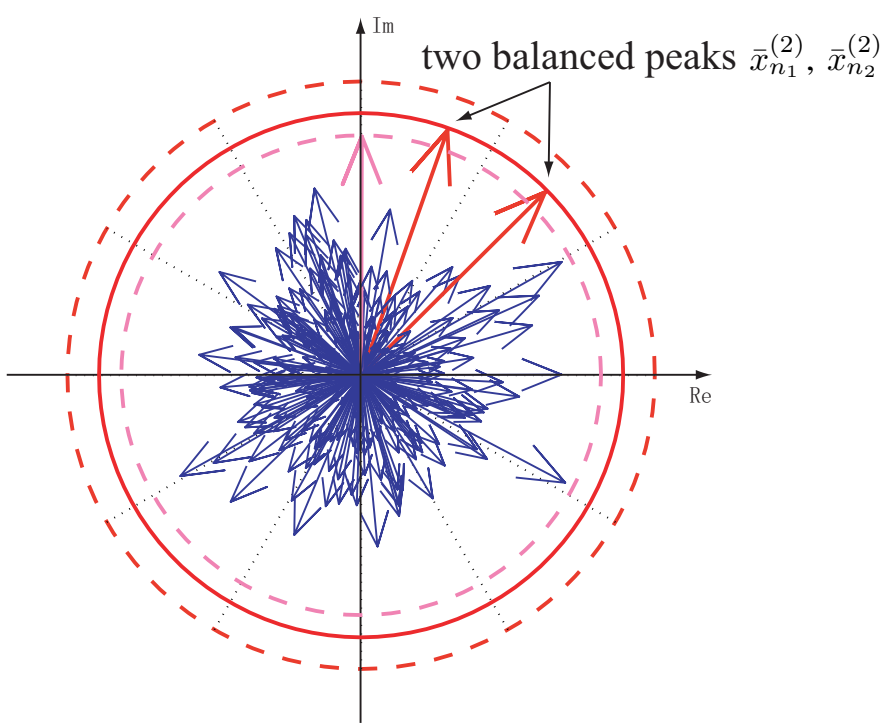

Fig. 3. The time-domain samples with two peaks balanced.

Mathematically, the algorithm can be written as

$$
\overline{\mathbf{x}}^{(i+1)}=\overline{\mathbf{x}}^{(i)}+\mu^{(i)} \mathbf{p}^{(i)}
$$

where $\mathbf{p}^{(i)}$ is the optimal clipping direction along which all balanced peaks can be reduced by identical magnitude. $\mu^{(i)}$ is the step-size at the $i$ th iteration such that a new peak is increased to the magnitude that is tangent to the shrunk circle. Thus, $\mathbf{p}^{(i)}$ and $\mu^{(i)}$ are two key qunatities that need to be determined at each iteration.

We begin with $i=2$ balanced peaks at locations $n_{1}$ and $n_{2}$. Given (16), the corresponding clipping impulses $\mathbf{p}_{n_{1}}^{(2)}$ and $\mathbf{p}_{n_{2}}^{(2)}$ can be easily obtained. Then the clipping direction $\mathbf{p}^{(2)}$ is the combination of $\mathbf{p}_{n_{1}}^{(2)}$ and $\mathbf{p}_{n_{2}}^{(2)}$, which can be written as

$$
\mathbf{p}^{(2)}=\beta_{n_{1}}^{(2)} \mathbf{p}_{n_{1}}^{(2)}+\beta_{n_{2}}^{(2)} \mathbf{p}_{n_{2}}^{(2)}
$$

Note that $\beta_{n_{1}}^{(2)}$ and $\beta_{n_{2}}^{(2)}$ are complex scalars rather than real ones, because the clipping impulse $\mathbf{p}_{n_{2}}^{(2)}$, for example, clips the peak at location $n_{2}$ and also changes the peak at location $n_{1}$. As illustrated in Fig.4, the $n_{1}$ th element of $\mathbf{p}_{n_{1}}^{(2)}$ denoted by $p_{n_{1} \rightarrow n_{1}}^{(2)}$ is opposite to the peak at location $n_{1}$, say $\bar{x}_{n_{1}}^{(2)}$. However, the $n_{1}$ th element of $\mathbf{p}_{n_{2}}^{(2)}$ denoted by $p_{n_{2} \rightarrow n_{1}}^{(2)}$ does not necessarily have the phase that is right opposite to $\bar{x}_{n_{1}}^{(2)}$. If $\beta_{n_{1}}^{(2)}$ and $\beta_{n_{2}}^{(2)}$ are real scalars, the combining contribution of $p_{n_{1} \rightarrow n_{1}}^{(2)}$ and $p_{n_{2} \rightarrow n_{1}}^{(2)}$ on $\bar{x}_{n_{1}}^{(2)}$ can be written as

$$
p_{n_{1}}^{(2)}=\beta_{n_{1}}^{(2)} p_{n_{1} \rightarrow n_{1}}^{(2)}+\beta_{n_{2}}^{(2)} p_{n_{2} \rightarrow n_{1}}^{(2)}
$$

which is not along the opposite direction of $\bar{x}_{n_{1}}^{(2)}$ except when $\beta_{n_{2}}^{(2)}=0$. Therefore, in order to clip the peak along its opposite direction, complex scalars are used to rotate the direction of $p_{n_{1}}^{(2)}$ such that

$$
\beta_{n_{1}}^{(2)} p_{n_{1} \rightarrow n_{1}}^{(2)}+\beta_{n_{2}}^{(2)} p_{n_{2} \rightarrow n_{1}}^{(2)}=-c_{n_{1}} \cdot \bar{x}_{n_{1}}^{(2)}
$$

where $c_{n_{1}}$ is a real constant. Similarly for another balanced peak $\bar{x}_{n_{2}}^{(2)}, \beta_{n_{1}}^{(2)}$ and $\beta_{n_{2}}^{(2)}$ need to satisfy

$$
\beta_{n_{1}}^{(2)} p_{n_{1} \rightarrow n_{2}}^{(2)}+\beta_{n_{2}}^{(2)} p_{n_{2} \rightarrow n_{2}}^{(2)}=-c_{n_{2}} \cdot \bar{x}_{n_{2}}^{(2)}
$$

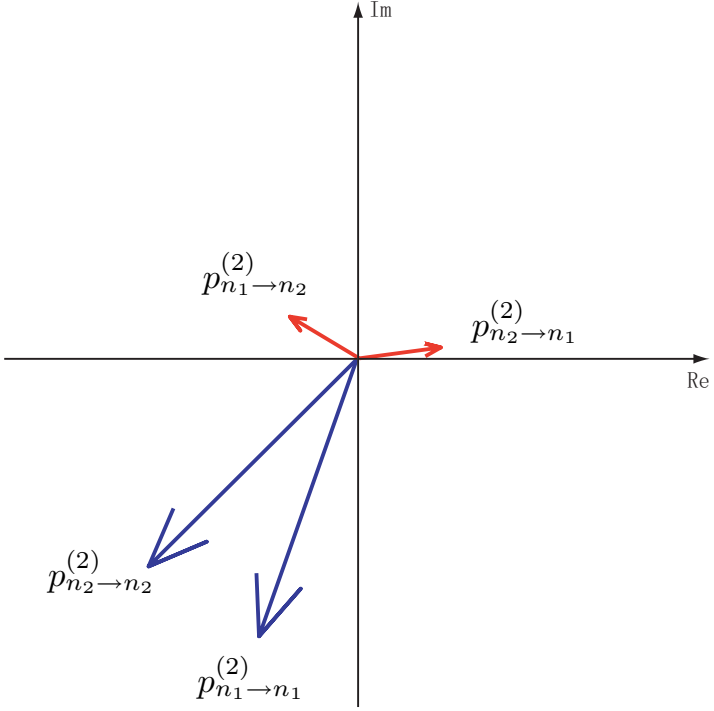

Fig. 4. The values of $\mathbf{p}_{n_{1}}^{(2)}$ and $\mathbf{p}_{n_{2}}^{(2)}$ at locations $n_{1}$ and $n_{2}$.

Note that $\beta_{n_{1}}^{(2)}$ and $\beta_{n_{2}}^{(2)}$ also determine the clipping magnitude on each peak. The clipping magnitude on both balanced peaks needs to be identical. Consequently, for a unit clipping magnitude, $\beta_{n_{1}}^{(2)}$ and $\beta_{n_{2}}^{(2)}$ can be found by solving

$$
\left\{\begin{array}{l}
\beta_{n_{1}}^{(2)} p_{n_{1} \rightarrow n_{1}}^{(2)}+\beta_{n_{2}}^{(2)} p_{n_{2} \rightarrow n_{1}}^{(2)}=-\bar{x}_{n_{1}}^{(2)} /\left|\bar{x}_{n_{1}}^{(2)}\right| \\
\beta_{n_{1}}^{(2)} p_{n_{1} \rightarrow n_{2}}^{(2)}+\beta_{n_{2}}^{(2)} p_{n_{2} \rightarrow n_{2}}^{(2)}=-\bar{x}_{n_{2}}^{(2)} /\left|\bar{x}_{n_{2}}^{(2)}\right|
\end{array}\right.
$$

For a general case with $i$ balanced peaks, the complex scalars can be calculated by solving

$$
\underbrace{\left(\begin{array}{cccc}
p_{n_{1} \rightarrow n_{1}}^{(i)} & p_{n_{2} \rightarrow n_{1}}^{(i)} & \cdots & p_{n_{i} \rightarrow n_{1}}^{(i)} \\
p_{n_{1} \rightarrow n_{2}}^{(i)} & p_{n_{2} \rightarrow n_{2}}^{(i)} & \cdots & p_{n_{i} \rightarrow n_{2}}^{(i)} \\
\vdots & \ddots & \ddots & \vdots \\
p_{n_{1} \rightarrow n_{i}}^{(i)} & p_{n_{2} \rightarrow n_{i}}^{(i)} & \cdots & p_{n_{i} \rightarrow n_{i}}^{(i)}
\end{array}\right)}_{D^{(i)}} \underbrace{\left(\begin{array}{c}
\beta_{n_{1}}^{(i)} \\
\beta_{n_{2}}^{(i)} \\
\vdots \\
\beta_{n_{i}}^{(i)}
\end{array}\right)}_{\boldsymbol{\beta}^{(i)}}=\gamma^{(i)}
$$

where $p_{n_{j} \rightarrow n_{k}}^{(i)}$ is the $n_{k}$ th element of the clipping impulse corresponding to the sample $\bar{x}_{n_{j}}^{(i)}$ and

$$
\gamma^{(i)}=\left(\begin{array}{c}
-\bar{x}_{n_{1}}^{(i)} /\left|\bar{x}_{n_{1}}^{(i)}\right| \\
-\bar{x}_{n_{2}}^{(i)} /\left|\bar{x}_{n_{2}}^{(i)}\right| \\
\vdots \\
-\bar{x}_{n_{i}}^{(i)} /\left|\bar{x}_{n_{i}}^{(i)}\right|
\end{array}\right)
$$

Now the clipping direction at the $i$ th iteration can be determined by

$$
\mathbf{p}^{(i)}=\sum_{j=1}^{i} \beta_{n_{j}}^{(i)} \mathbf{p}_{n_{j}}^{(i)}
$$

An interesting feature of the matrix $D^{(i)}$ in (24) is that it converges to a singular matrix quickly, as $i$ increases. Thus, the algorithm is capable of reducing most of the possible PAR reduction after very few iterations. This will be shown in the simulation study included in the following section. Another 
consequence is that one does not need to compute the inverse of large matrices in practice.

Given the clipping direction $\mathbf{p}^{(i)}$, the next step is to determine the clipping step-size $\mu^{(i)}$. Since a unit step-size is used for computing $\boldsymbol{\beta}^{(i)}$ in (24), $\mu^{(i)}$ is essentially the amount that the radius of the circle can be reduced at the $i$ th iteration. In our approach, we use $\mu^{(i)}=a_{i} r^{(i-1)}$ where $a_{i}$ is a real number between 0 and 1 . Thus, the test radius becomes $r_{t}^{(i)}=\left(1-a_{i}\right) r^{(i-1)}$. Beginning with this step-size, we can determine $\mu^{(i)}$ as follows:

1) Compute $\overline{\mathbf{x}}^{(i+1)}$ with the step-size $\mu^{(i)}=a_{i} r^{(i-1)}$. Check all samples of $\overline{\mathbf{x}}^{(i+1)}$ to see if the magnitude of any sample is greater than the test radius $r_{t}^{(i)}$. Record the indexes of these samples to a set $B^{(i)}$ as candidate samples for the next balanced peak. The next balanced peak must be one sample in set $B^{(i)}$. If $B^{(i)}=\emptyset$, increase $a_{i}$ until $B^{(i)} \neq \emptyset$.

2) For any sample $\bar{x}_{b}^{(i)}$ contained in $B^{(i)}$, we compute the associated step-size $\mu_{b}^{(i)}$ such that the peak of this sample is balanced with the circle, i.e.,

$$
r^{(i-1)}-\mu_{b}^{(i)}=\left|\bar{x}_{b}^{(i)}+\mu_{b}^{(i)} p_{b}^{(i)}\right|, \quad \mu_{b}^{(i)}>0
$$

where $p_{b}^{(i)}$ is the $b$ th element of $\mathbf{p}^{(i)}$. If (27) has two positive roots, then $\mu_{b}^{(i)}$ is the smaller positive root. The clipping step-size $\mu^{(i)}$ is determined by

$$
\mu^{(i)}=\min _{b \in B^{(i)}} \mu_{b}^{(i)}
$$

When choosing $r_{t}^{(i)}$, we need to take into account that: if $r_{t}^{(i)}$ is too large, we have too many candidate samples to consider; if $r_{t}^{(i)}$ is too small, we obtain no candidate samples ${ }^{2}$. Since we generally expect the radius to shrink monotonically on the average, the radius reduction in the previous iteration can be used for setting the initial test radius at the $i$ th iteration, i.e.,

$$
r_{t}^{(i)}=\left(1-a_{i}\right) r^{(i-1)}=r^{(i-1)}-\mu^{(i-1)}
$$

\section{Complexity analysis}

At the $i$ th iteration, $i$ approximate impulses need to be computed corresponding to $i$ balanced peaks by (16), where $-\frac{1}{L} Q_{c} q_{n}^{H}$ can be computed in advance and stored in a lookup table. Computing the approximate impulses requires $i(N L+1)$ complex multiplications. Given approximate impulses $\left\{\mathbf{p}_{n}\right\}$, the complex scalars $\boldsymbol{\beta}^{(i)}$ can be calculated by the complex-valued linear equations described in (24). It is equivalent to solving a real-valued $2 i \times 2 i$ system of equations. Note that the arithmetic complexity of solving a general $2 i \times 2 i$ system of equations is $O\left(8 i^{3}\right)$ [11]. Fortunately, a key point is that only few iterations are needed to achieve good performance. Thus, one only needs to solve a small-scale system of equations at each iteration. Additional $(i-1) N L$ complex additions and $i N L$ complex multiplications are needed for forming the clipping direction $\mathbf{p}^{(i)}$ via (26). In the step-size

\footnotetext{
${ }^{2}$ The role of $r_{t}^{(i)}$ is akin to that of the search radius in sphere decoding algorithm [10]
}

computation, initial clipping and full search over all samples require $2 N L$ complex additions and complex multiplications for the candidate samples. We ignore the computation for solving (27) since the number of candidate samples $b^{(i)} \ll$ $N L$.

The overall computational complexity of the proposed algorithm for a total number of $N_{I}$ iterations is $\frac{1}{2}\left(N_{I}^{2}+1\right) N L$ complex additions, $\left(N_{I}^{2}+N_{I}\right)\left(N L+\frac{1}{2}\right)$ complex multiplications and extra $\sum_{i=1}^{N_{I}} O\left(8 i^{3}\right)$ operations. Thus, our algorithm has a complexity that is linear in $N L$. By comparison, the complexity for solving the QCQP in (8) by the interiorpoint method is $O\left((N L)^{1 / 2}\left(N L+N_{r}\right) N_{r}^{2}\right)$ [12], i.e., proportional to $(N L)^{3 / 2}$. Therefore, the proposed algorithm can reduce the computational complexity significantly and achieve near-optimal performance, as shown in the simulation section.

\section{Simulation RESUlts}

In this section, simulation results are presented to document the performance of the algorithm proposed in Section III. We consider an OFDM system with $N=256$ subcarriers, in which 10 subcarriers are randomly chosen for reserved tones. QAM modulation is used in the remaining subcarriers for data transmission. We assume Nyquist sampling $(L=1)$, for simplicity.

To evaluate the PAR reduction performance, we compare the complementary cumulative distribution functions (CCDFs) achieved by the proposed algorithm with a different number of iterations. The results are illustrated in Fig.5. The CCDFs represent the probability that the system PAR is greater than a specific threshold. Also for comparison, we show the CCDFs for the case where tone reservation is performed optimally by QCQP and the case with no PAR reduction. It can be seen that the proposed algorithm can reduce the PAR significantly even with 2 or 3 iterations. With more iterations, the PAR reduction performance converges. In Fig.5, indeed, the performance difference between the algorithm with ten iterations and the one with ten more iterations is not noticeable. We observe that the converged performance is very close to the optimal performance that is achieved by solving the QCQP exactly at considerable computational cost. We can conclude that near-optimal performance can be achieved by the proposed algorithm with much lower computational complexity than QCQP.

\section{CONCLUSIONS}

We have presented an iterative clipping algorithm for complex-baseband tone reservation. At each iteration, the optimal clipping direction is computed such that all balanced peaks can be clipped by identical magnitude, which leads to fast convergence and no peak regrowth. Simulation results show that the presented algorithm can achieve near-optimal performance with low computational cost.

\section{REFERENCES}

[1] R. Van Nee and R. Prasad. OFDM for Wireless Multimedia Communications. Artech House, 2000. 


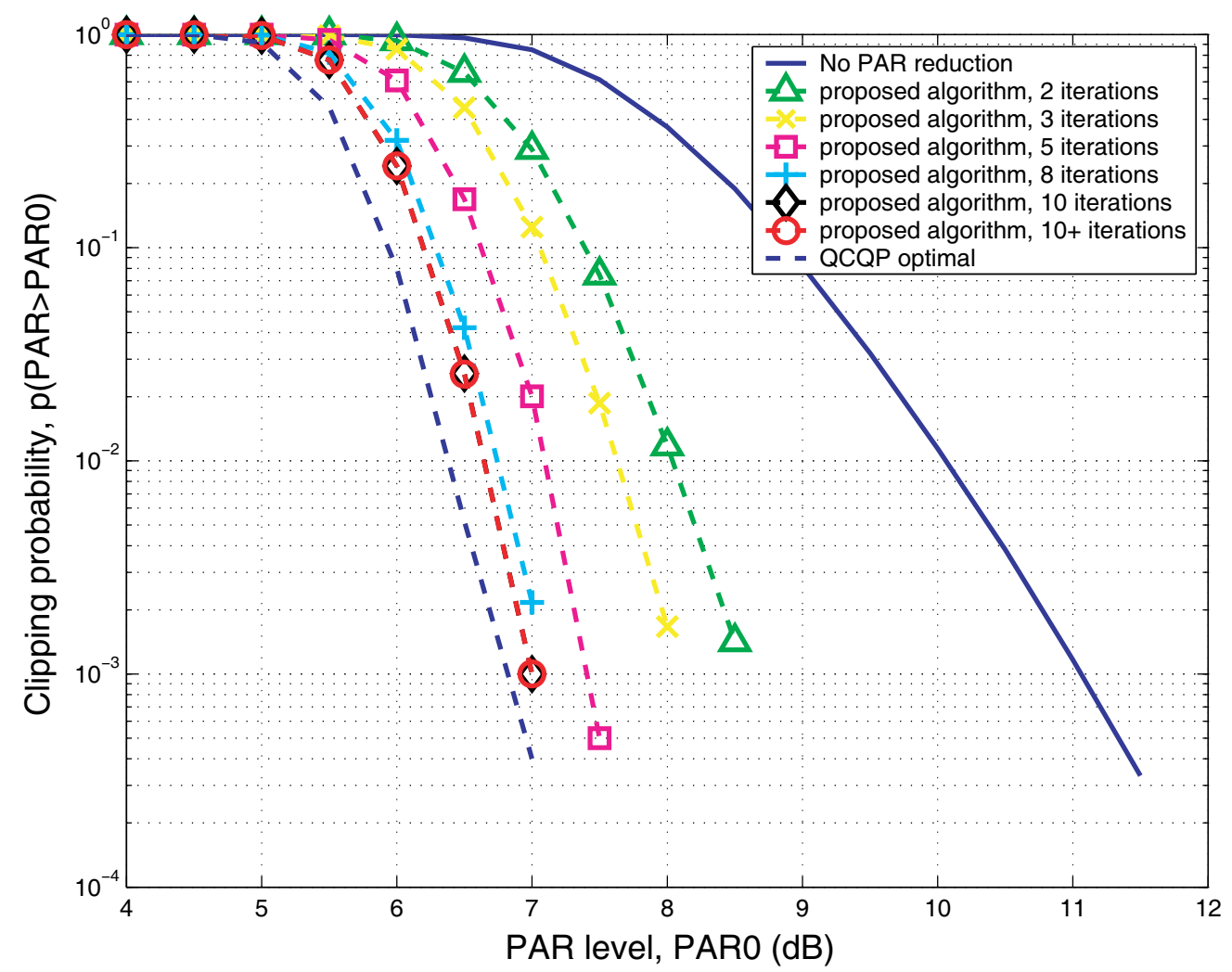

Fig. 5. Performance of the proposed algorithm with different iterations.

[2] Supplement to IEEE Std. 802.11, Wireless LAN Media Access Control (MAC) and Physical Layer (PHY) Specifications: High-Speed Physical Layer in the $5 \mathrm{GHZ}$ Band. IEEE Standard 802.11a, 2001.

[3] Broadband Radio Access Networks (BRAN): HIPERLAN Type 2 Physical (PHY) layer. ETSI TS 101475 V1.1.1, 2000.

[4] IEEE Std 802.16e-2005, "IEEE Standard for Local and Metropolitan Area Networks Part 16: Air Interface for Fixed Broadband Wireless Access Systems", February 2006.

[5] Seung Hee Han and Jae Hong Lee. An overview of peak-to-average power ratio reduction techniques for multicarrier transmission. IEEE Wireless Communications, 12(2):56-65, April 2005.

[6] J. Tellado-Mourelo. Peak to average power reduction for multicarrier modulation. PhD thesis, Stanford University, 1999.

[7] B. S. Krongold and D. L. Jones. An active-set approach for OFDM PAR reduction via tone reservation. IEEE Transactions on Signal Processing, 52(2):495-509, February 2004.

[8] A. T. Erdogan. A low complexity multicarrier PAR reduction approach based on subgradient optimization. Signal Processing, 86:3890-3903, 2006.

[9] M. Wang, D. E. Quevedo, G. C. Goodwin, and B. S. Krongold. OFDMA uplink PAR reduction via tone reservation. In IEEE Global Telecommunications Conference, GLOBECOM '07, Washington, D. C., 2007.

[10] B. Hassibi and H. Vikalo. On the sphere-decoding algorithm I. Expected complexity. IEEE Transactions on Signal Processing, 53:2806-2818, August 2005

[11] G. H. Golub and C. F. Van Loan. Matrix Computations, 3rd Ed. The Johns Hopkins University Press, Baltimore, MD, 1996.

[12] A. Nemirovski. Interior Point Polynomial Time Methods in Convex Programming. http://www2.isye.gatech.edu/nemirovs/, 2004. 